\title{
Both structure and function of human monoclonal antibodies contribute to enhancement of Zika virus infectivity in vitro
}

\author{
Min $\mathrm{Li}^{1,2}$, Xin Wang ${ }^{1,2}$, Qihui Wang ${ }^{3}$, Lei Yu ${ }^{4}$, Lanfeng Wang ${ }^{1,2}$, Jinghua Yan ${ }^{3}$, Fuchun Zhang ${ }^{4}$, \\ Linqi Zhang ${ }^{5}$, George F. Gao ${ }^{2,3} \&$ Xia Jin ${ }^{1,2^{*}}$ \\ ${ }^{1}$ CAS Key Laboratory of Molecular Virology and Immunology, Institut Pasteur of Shanghai, Shanghai 200031, China; \\ ${ }^{2}$ University of Chinese Academy of Sciences, Beijing 100049, China; \\ ${ }^{3}$ CAS Key Laboratory of Microbial Physiological and Metabolic Engineering, Institute of Microbiology, Chinese Academy of Sciences, \\ Beijing 100101, China; \\ ${ }^{4}$ Guangzhou Eighth People's Hospital, Guangzhou Medical University, Guangzhou 510060, China; \\ ${ }^{5}$ Comprehensive AIDS Research Center, Collaborative Innovation Center for Diagnosis and Treatment of Infectious Diseases, School of \\ Medicine, Tsinghua University, Beijing 100084, China
}

Received August 2,2017; accepted September 11,2017; published online November 10, 2017

Citation: $\quad$ Li, M., Wang, X., Wang, Q., Yu, L., Wang, L., Yan, J., Zhang, F., Zhang, L., Gao, G.F., and Jin, X. (2017). Both structure and function of human monoclonal antibodies contribute to enhancement of Zika virus infectivity in vitro. Sci China Life Sci 60, 1396-1398. https://doi.org/10.1007/s11427017-9192-0

Dear Editor,

Antibody-dependent enhancement (ADE) has long been recognized for dengue virus (DENV) in vitro and in vivo. It is now clear that antibodies to DENV can also enhance Zika virus (ZIKV) infection in vitro, and vice versa (Dejnirattisai et al., 2016; Stettler et al., 2016). The characteristics of enhancing antibodies, however, remain elusive. Some have suggested that non-neutralizing antibodies or antibodies recognizing specific antigenic sites are enhancing antibodies: such as those that bind to prM of dengue virus. But others showed that even highly potent neutralizing murine antibodies could enhance West Nile virus (WNV) and ZIKV infection in vitro at extremely low concentrations (Pierson et al., 2007). Thus, neutralization and enhancement might not be dichotomous results generated by different antibodies, but divergent outcomes of immune response mediated by the same antibody. Because ADE is implicated in pathogenesis of ZIKV infection and it causes complications

*Corresponding author (email: xjin@ips.ac.cn) in vaccine design and testing, we sought to determine the characteristics of antibodies capable of enhancing ZIKV infectivity using 14 newly discovered ZIKV specific human monoclonal antibodies (mAbs).

Fourteen ZIKV specific human mAbs that target DIII (Z23, Z30, ZK2B10), DI/DII (Z20, Z3L1, ZK7C3, ZK8-4, ZK12G2, ZK12-2), DI/DII/DIII (ZK2C2, ZK1G4) or fusion loop (FL: Z5, Z6, Z7) of E protein were isolated from three Chinese patients who had returned from endemic countries with a confirmed ZIKV infection (Wang et al., 2016; Yu et al., 2017), and two isotype matched control human $\mathrm{mAbs}$ that recognize Ebola virus (EBOV) glycoprotein (2G4) and Middle East respiratory syndrome coronavirus (MERS-CoV) S protein (Mers-4), respectively, were included in this study (Table S1 in Supporting Information). All ZIKV mAbs included in this study bound to ZIKV E protein with varied affinity as determined by ELISA, and some of them cross-reacted with DENV-1 and DENV-2 E proteins. These $\mathrm{mAbs}$ neutralized $\mathrm{ZIKV}$ at a range of $\mathrm{IC}_{50}$ from as high as $0.04 \mu \mathrm{g} \mathrm{mL}^{-1}$ to as low as more than $40 \mu \mathrm{g} \mathrm{mL}^{-1}$; again, some of which also neutralized DENV-1 and DENV-2. Among them, four of the most potent neutralizing mAbs that 
targeted DI/DII (Z20, Z3L1) or DIII (Z23, ZK2B10) were tested in vivo for protection against ZIKV challenge in an adoptive transfer model using AG6 type I interferon deficient mice. Three of these antibodies (3/4) demonstrated 100\% protection, and one of them (1/4) showed $80 \%$ protection (Table S1 in Supporting Information) (Wang et al., 2016; $\mathrm{Yu}$ et al., 2017). Hence, domain-specificity appears not to be an absolute determinant of antibody-mediated protection against virus infection.

We assessed the ability of these 14 ZIKV specific human mAbs to enhance ZIKV infection with K562 cells using a method described in recent papers (Dejnirattisai et al., 2016; Stettler et al., 2016). As shown by a representative assay in Figure 1A, various dilutions of ZIKV antibodies (ZK2B10 and ZK8-4) or control mAb Mers-4 were incubated with ZIKV first, and then added to K562 cells. The cells were incubated at $37^{\circ} \mathrm{C}$ for $48 \mathrm{~h}$, and then harvested, intracellularly stained with an anti-E antibody, and then subjected to flow cytometry analysis. Results showed that peak enhancement levels were reached at $0.8 \mu \mathrm{g} \mathrm{mL}^{-1}$ for ZK2B10 and $100 \mu \mathrm{g} \mathrm{mL}^{-1}$ for $\mathrm{ZK} 8-4$, at which $23.3 \%$ and $18.7 \%$ cells were infected. In the absence of these mAbs, there were only $0.28 \%$ cells positive for ZIKV antigen staining. These results correspond to an approximately 83- and 67-fold enhancement by comparison to the percentage of ZIKV infected cells in the absence of antibodies (Figure 1A). When all 14 mAbs were examined together, 2/3 FL specific, 5/6 EDI/DII specific, 3/3 EDIII specific, and 1/2 EDI/DII/DIII specific mAbs enhanced ZIKV infectivity for more than 20-fold (Figure 1B). Two mAbs in EDI/II group (Z20 and Z3L1) exhibited the highest enhancing activities, approximately 300-fold, of ZIKV infection, whereas the remaining four mAbs targeting EDI/II displayed modest to low enhancement capacities. Thus, domain-specificity alone is insufficient to determine whether an antibody is enhancing or not. Notably, the logarithmic values of peak $\mathrm{ADE}$ antibody concentration and the logarithmic $\mathrm{IC}_{50}$ values of neutralization concentration showed a significant linear correlation $(R=0.80$, $P=0.0007$ ) (Figure 1C).

Our results generated in this study are consistent with a recent paper showing that human antibodies against ZIKV EDI/II were cross-reactive, poorly neutralizing, whereas
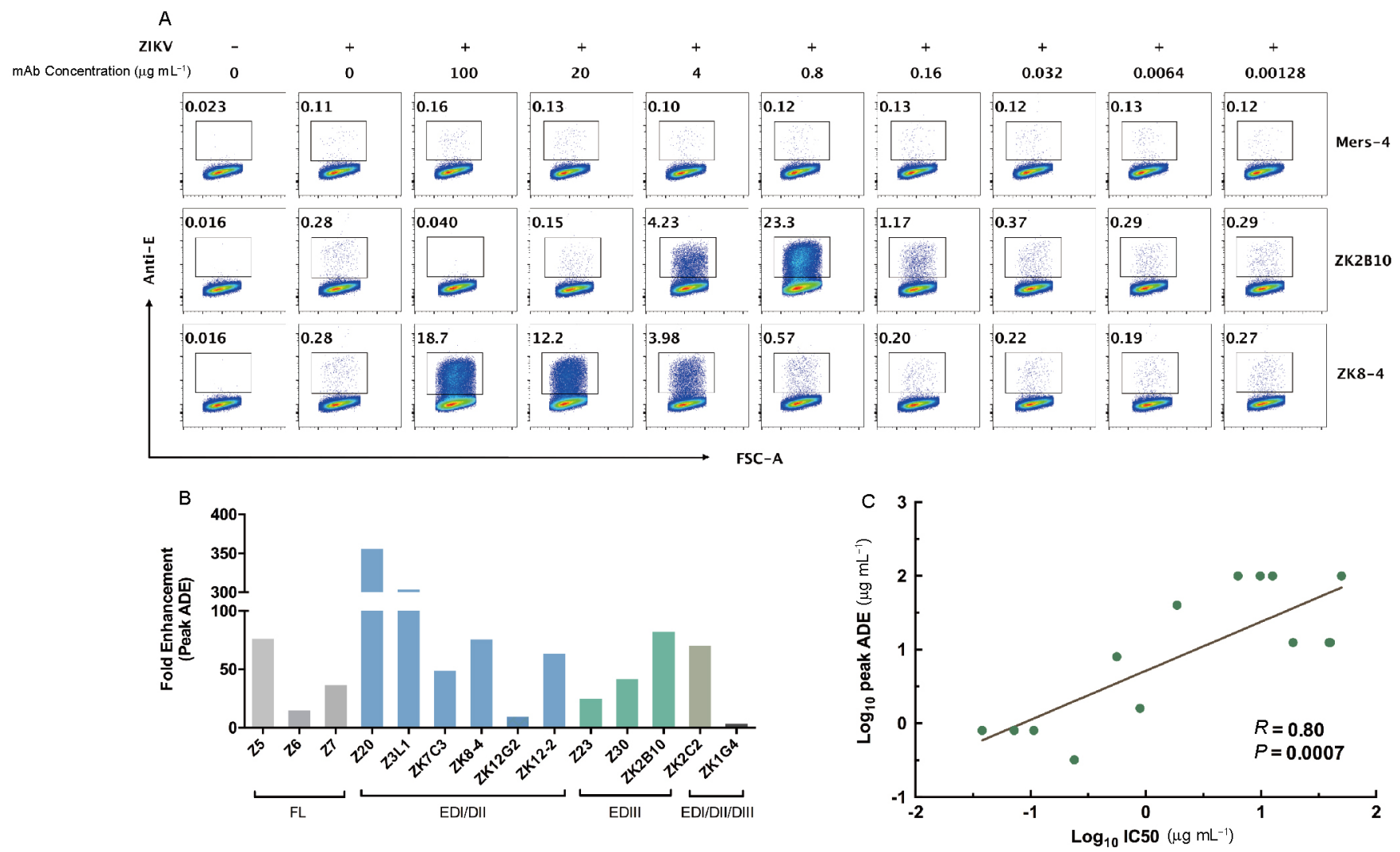

Figure 1 The structural and functional basis of mAbs capable of enhancing ZIKV infection in vitro. A, K562 cells were infected with ZIKV in the presence or absence of serially diluted human monoclonal antibodies Mers-4 (anti-MERS-Co), ZK2B10 (anti-ZIKV-EDIII) and ZK8-4 (anti-ZIKV-DI/DII). Cells were harvested and stained intracellularlly with a pan-flavivirus antibody 4G2, and then analyzed by flow cytometry. B, Fold enhancement was analyzed by comparison to the percentage of infected cells in the presence or absence of antibodies. Antibodies were classified into four groups according to their binding sites on ZIKV soluble envelope protein. FL, fusion loop; EDI, domain I of envelope protein; EDII, domain II of envelope protein; EDIII, domain III of envelope protein. C, The $\log _{10} \mathrm{IC}_{50}$ value was plotted against the corresponding $\log _{10}$ concentration of antibody at which the peak ADE was reached for ZIKV ( $n=14$ ) infections. The $R$ value and $P$ value were calculated by linear regression analysis using GraphPad Prism 6.0 software. 
those targeted EDIII or quaternary epitopes were potent neutralizing; however, after being diluted, they could all enhance ZIKV infectivity in K562 cells (Stettler et al., 2016). Thus, epitope-specificity per se is not a sufficient determinant of whether an antibody will enhance infection or not, at least in vitro. In fact, even highly neutralizing antibodies that can enhance virus infection have also been documented previously (Pierson et al., 2007). Using a panel of 6 West Nile virus (WNV) EDIII specific murine mAbs that had an $\mathrm{EC}_{50}$ ranging from 0.018 to $39.5 \mathrm{nmol} \mathrm{L}^{-1}$, it was convincingly demonstrated that all neutralizing antibodies could enhance the infection of WNV reporter virus particles in K562 cells (Pierson et al., 2007). Based on our and other published data using flavivirus specific mAbs, it can be concluded that neutralization and enhancement are two linked properties of the same antibody. This conclusion is consistent with a generally accepted "Occupancy theory" of antibody-virus interaction.

Importantly, we provided the first demonstration of a quantitative association between the concentrations for neutralization and peak enhancement for the same antibody (Figure 1C). By taking advantage of a panel of human monoclonal antibodies against various domains of ZIKV E protein, we further revealed a straightforward correlation between antibody-mediated neutralization and enhancement among flaviviruses. These findings provided answer to a long standing theoretical puzzle regarding the principal characteristics of an enhancing antibody: it is its function, not just structure. Our results also suggest a simple assay for predicting the ADE potential of a neutralizing antibody, and thus supplementing the current methods for assessing the beneficial and adverse effects of antibodies elicited by vaccination.
Compliance and ethics The author(s) declare that they have no conflict of interest.

Acknowledgements The work was supported by National Key Program Project Grant of Ministry of Science and Technology of China (2016YFC1201000), the Strategic Priority Research Program of the Chinese Academy of Sciences (XDBP 030405) and grants from the Municipal Science and Technology Bureau Foundation of Guangzhou (2014Y2-00550, 201508020263).

Dejnirattisai, W., Supasa, P., Wongwiwat, W., Rouvinski, A., Barba-Spaeth, G., Duangchinda, T., Sakuntabhai, A., Cao-Lormeau, V.M., Malasit, P., Rey, F.A., Mongkolsapaya, J., and Screaton, G.R. (2016). Dengue virus sero-cross-reactivity drives antibody-dependent enhancement of infection with zika virus. Nat Immunol 17, 1102-1108.

Pierson, T.C., Xu, Q., Nelson, S., Oliphant, T., Nybakken, G.E., Fremont, D.H., and Diamond, M.S. (2007). The stoichiometry of antibody-mediated neutralization and enhancement of West Nile virus infection. Cell Host Microbe 1, 135-145.

Stettler, K., Beltramello, M., Espinosa, D.A., Graham, V., Cassotta, A., Bianchi, S., Vanzetta, F., Minola, A., Jaconi, S., Mele, F., Foglierini, M., Pedotti, M., Simonelli, L., Dowall, S., Atkinson, B., Percivalle, E., Simmons, C.P., Varani, L., Blum, J., Baldanti, F., Cameroni, E., Hewson, R., Harris, E., Lanzavecchia, A., Sallusto, F., and Corti, D. (2016). Specificity, cross-reactivity, and function of antibodies elicited by Zika virus infection. Science 353, 823-826.

Wang, Q., Yang, H., Liu, X., Dai, L., Ma, T., Qi, J., Wong, G., Peng, R., Liu, S., Li, J., Li, S., Song, J., Liu, J., He, J., Yuan, H., Xiong, Y., Liao, Y., Li, J., Yang, J., Tong, Z., Griffin, B.D., Bi, Y., Liang, M., Xu, X., Qin, C., Cheng, G., Zhang, X., Wang, P., Qiu, X., Kobinger, G., Shi, Y., Yan, J., and Gao, G.F. (2016). Molecular determinants of human neutralizing antibodies isolated from a patient infected with Zika virus. Sci Transl Med 8, 369ra179-369ra179.

Yu, L., Wang, R., Gao, F., Li, M., Liu, J., Wang, J., Hong, W., Zhao, L., Wen, Y., Yin, C., Wang, H., Zhang, Q., Li, Y., Zhou, P., Zhang, R., Liu, Y., Tang, X., Guan, Y., Qin, C.F., Chen, L., Shi, X., Jin, X., Cheng, G., Zhang, F., and Zhang, L. (2017). Delineating antibody recognitio$\mathrm{n}$ against Zika virus during natural infection. JCI Insight in press doi: 10.1172/jci.insight.93042.

\section{SUPPORTING INFORMATION}

Table S1 Characteristics of human monoclonal antibodies used in the study

The supporting information is available online at http://life.scichina.com and https://link.springer.com. The supporting materials are published as submitted, without typesetting or editing. The responsibility for scientific accuracy and content remains entirely with the authors. 